\title{
REESTRUCTURACIÓN PRODUCTIVA E INTEGRACIÓN. TLCAN 20 AÑOS DESPUÉS
}

\begin{abstract}
Clemente Ruiz ${ }^{*}$
Fecha de recepción: 1 de julio de 2014. Fecha de aceptación: 27 de octubre de 2014.

RESUMEN

Con la entrada en vigor del Tratado de Libre Comercio de América del Norte (TLCAN), se crearon expectativas de alcanzar un mayor crecimiento, un aumento en el empleo y una mejora en los niveles de vida en México, Estados Unidos y Canadá. Veinte años después si bien se ha logrado una progresiva integración productiva, los efectos han sido sumamente diferenciados, sin que se haya observado una convergencia en los niveles de bienestar. En este artículo se plantea que la parte más positiva de la relación de México con los miembros del TLCAN ha sido, sin lugar a dudas, el desarrollo de cadenas globales de valor, las cuales se han desarrollado a partir de un crecimiento espectacular en el comercio trilateral. Finalmente, se ofrecen algunas reflexiones sobre los aspectos que deben considerarse para el futuro del Tratado, a fin de construir relaciones económicas y sociales más sólidas y benéficas para los tres países.
\end{abstract}

Palabras clave: intercambio comercial, integración productiva, cadenas globales de valor, inversión extranjera, empleo.

Clasificación JEL: E24, F13, F14, F15, F21, F55, O47.

\section{Productive Restructuring and Integration: NAFTA, 20 YEARS LATER}

\begin{abstract}
When the North American Free Trade Agreement (NAFTA) entered into force, there were expectations of stronger growth, more jobs and improved living conditions in Mexico, the United States and Canada. Twenty years later, although progressive productive integration has been achieved, its effects have been tremendously disparate, and welfare standards have diverged. This article proposes that the most positive aspect of the relationship between Mexico and other NAFTA members has, undoubtedly, been the development of global value chains, which emerged from enormous growth in trilateral trade. Finally, this work offers some reflections to bear in mind for the future of the agreement, aiming to build more solid and mutually beneficial economic and social relationships among the three countries.
\end{abstract}

Key Words: Commercial exchange, productive integration, global value chains, foreign investment, jobs.

* Facultad de Economía de la unAm, México. Correo electrónico: ruizdc@unam.mx 


\section{RESTRUCTURATION PRODUCTIVE ET INTÉGRATION. TLCAN 20 ANS APRÈS. Résumé}

Avec l'entrée en vigueur de l'Accord de libre échange nord-américain (TLCAN en espagnol) sont nés des expectatives de parvenir à une croissance majeure, la création d'emplois et une amélioration du niveau de vie au Mexique, aux États-Unis et au Canada. Vingt ans après, si une progressive intégration productive a été atteinte, les effets ont été extrêmement différents d'un pays à l'autre, sans qu'on ait pu observer une convergence en ce qui concerne la qualité de vie. Dans cet article, il est posé que l'aspect le plus positif de la relation du Mexique avec les autres membres du TLCAN a été, sans aucun doute, le développement de chaines globales de valeur, lesquelles ont été le produit d'une croissance spectaculaire du commerce trilatéral. Finalement, quelques réflexions sont présentées concernant les aspects qui doivent être pris en compte dans le futur de l'Accord, afin de construire des relations économiques et sociales plus solides et bénéfiques pour les trois pays.

Mots clés: échange commercial, intégration productive, chaines globales de valeur, investissement étranger, emploi.

\section{RESTRUTURAÇÁOO PRODUTIVA E INTEGRAÇÁO. TLCAN 20 ANOS DEPOIS Resumo}

Com a entrada em vigor do Tratado de Livre Comércio da América do Norte (TLCAN), se criaram expectativas de alcançar um maior crescimento, um aumento no emprego e uma melhoria nos níveis de vida no México, Estados Unidos e Canadá. Vinte anos depois, apesar de que se logrou uma progressiva integraçáo produtiva, os efeitos foram sumamente diferenciados, sem que se tenha observado uma convergência nos níveis de bem-estar. Neste artigo se concebe que a parte mais positiva da relação do México com os membros do TLCAN foi, sem lugar a dúvidas, o desenvolvimento de cadeias globais de valor, as quais se desenvolveram a partir de um crescimento espetacular no comercio trilateral. Finalmente, se oferecem algumas reflexóes sobre os aspectos que se devem considerar para o futuro do Tratado, a fim de construir relaçôes econômicas e sociais mais solidas e benéficas para os três países.

Palavras-chave: intercâmbio comercial, integração comercial, integraçáo produtiva, cadeia globais de valor, investimento estrangeiro, emprego.

\section{生产重组和一体化：北美自由贸易协定20年之后}

摘要：北美自由贸易协定生效之后，墨西哥、美国和加拿大都预期能够实 现高速增长, 创造更多的就业和提高生活条件。二十年之后，尽管生产领 域的一体化取得了很大进展, 但自贸协定所产生的效应与预期还有很大差 距disparate，而且三国的福利水平差距也在扩大。本文认为，墨西哥与美 国和加拿大在北美自由贸易框架内最为积极的一面毫无疑问就是墨西哥的 产业融入了全球价值链, 主要是通过三国之间的贸易快速增长实现的。最 后，本文的研究对北美自由贸易协定的重新谈判提供了一些思考，目的是 在三个成员国之间构建更加牢固、共同的经济和社会关系。 关键词：贸易交换 生产一体化 全球价值链 外国投资就业 


\section{INTRODUCCIÓN ${ }^{1}$}

En agosto de 1992, las negociaciones del Tratado de Libre Comercio entre México, Estados Unidos y Canadá (Tratado de Libre Comercio de América del Norte, TLCAN) fueron concluidas. En su momento, este acuerdo fue el más ambicioso que se realizaba entre un país en desarrollo y dos economías plenamente desarrolladas. El Tratado contenía disposiciones innovadoras, que sirvieron como modelo para la nueva generación de acuerdos de libre comercio durante los ańos noventa y principios del siglo xxi. Dada la trascendencia del suceso, el entonces presidente de México, Carlos Salinas de Gortari, dio un discurso a la nación y planteó que las ventajas y retos que traería al país la firma del TLCAN serían los siguientes: i) México se convertiría en "puente" de América Latina con Estados Unidos, "uno de los centros de la economía mundial", ii) se garantizaría un "acceso amplio y permanente" de los productos nacionales al enorme mercado estadounidense, con efectos esperados favorables para las empresas derivados de las economías de escala, al generar empleos, reducir sus costos, y dotándolas de mayor eficiencia, iii) mayor certeza jurídica para el comercio con los países socios, iv) especialización en sectores en los que México tenía ventajas, con la posibilidad de tener acceso a más tecnologías, $v$ ) beneficios para los consumidores en términos de una gama más amplia de productos, mayor calidad y precios más bajos, vi) incremento en las inversiones en México, que se traduciría en una mayor generación de empleos mejor remunerados, factores que en última instancia permitirían crecer a ritmos más elevados.

Finalmente, el TLCAN fue aprobado por los congresos de los tres países y entró en vigor en enero de 1994. Valoraciones de los beneficios del TLCAN tras cumplir su primer década, sugerían ya que muchos de los mencionados beneficios pronosticados no se habían cumplido, principalmente en términos del crecimiento económico elevado y sostenido, las inversiones en capital fijo y la generación de empleos (Moreno-Brid et al., 2006; Blecker, 2005; Calva, 2004). Veinte años han pasado desde su entrada en vigor y sus efectos han sido decisivos para la modificación de la estructura productiva de los tres países signantes. En este artículo se plantea que, en realidad, con el TLCAN aconteció

1 Una versión previa fue presentada en el seminario "20 años del Tratado de Libre Comercio de América del Norte: viejos problemas nuevos desafíos”, realizada en el Departamento de Estudios de Posgrado de la Facultad de Economía de la unam, 23 y 24 de enero de 2014.

2 Mensaje que el presidente de México, Carlos Salinas de Gortari, dirigió a la nación con motivo del fin de las negociaciones del TLCAN el 12 de agosto de 1992. 
una larga transición hacia la integración productiva de las tres economías, pero con importantes asimetrías.

\section{EL PROCESO DE CRECIMIENTO ECONÓMICO EN UN ENTORNO DE INTEGRACIÓN}

La expectativa en los años noventa era que el TLCAN elevaría el ritmo de crecimiento económico de los tres países. Tras dos décadas existe evidencia de que esto no aconteció. Analizando un largo periodo -desde los años sesenta hasta la actualidad-, podemos observar que la fase de fuerte crecimiento ocurrió de 1960 a 1981, en donde lo acontecido se apegó a la teoría del crecimiento económico, que arguye que los países con un menor grado de desarrollo son los que observan un mayor crecimiento, y que en la medida que se desarrollan este ritmo se reduce, igualándose con el observado en los países industrializados (Kuznets, 1956). En 1982, con la aparición de la crisis de la deuda, el crecimiento de los tres países se redujo, afectando especialmente a México, tal como se muestra en el cuadro 1. En los años noventa, con la entrada en vigor del TLCAN, la expectativa era que México crecería a un ritmo mayor; sin embargo, lo que se ha observado es sólo un pequeño incremento en la tasa de crecimiento, con una tendencia hacia la homogenización de las tasas entre los países de la región. Se podría sugerir que lo que aconteció fue una sincronización del ritmo de crecimiento en alrededor del 2.5\% promedio anual.

Un bajo crecimiento económico para un país en desarrollo como México, implica la imposibilidad de cerrar la brecha de ingreso con los principales socios comerciales (Blecker y Esquivel, 2013); se observó en México que mientras su ritmo de crecimiento menguaba, su población continuaba aumentando de forma importante. En el periodo 1960 a 1981, la tasa de crecimiento económico fue de $6.8 \%$, lo que permitía reducir la brecha del producto interno bruto (PIB) por habitante con Estados Unidos y Canadá. En el periodo subsiguiente, de 1982 a 1993, la población en México creció a un ritmo anual de $2 \%$, es decir, por encima del crecimiento económico, lo que llevó a un incremento de la desigualdad con respecto a Estados Unidos y Canadá. De 1994 a 2013, la tasa de crecimiento de la población disminuyó a $1.5 \%$, pero el crecimiento económico no era suficiente para cerrar la brecha de ingreso, dado que el diferencial entre el crecimiento de la economía y el de la población fue de 1.2\% para México, de 1.6 para Estados Unidos y de 1.7 para el caso de Canadá. Esto significó que, lejos de reducirse, la desigualdad continuó intensificándose, tal como se muestra en la gráfica 1 . 
Cuadro 1. México, Canadá y Estados Unidos: periodos de crecimiento económico, 1960-2013 (tasa de crecimiento promedio anual del PIB)

\begin{tabular}{lccc}
\hline & Canadá & Estados Unidos & México \\
\hline 1960 a 1981 & $4.8 \%$ & $3.8 \%$ & $6.8 \%$ \\
1982 a 1993 & $2.5 \%$ & $3.5 \%$ & $2.0 \%$ \\
1994 a 2013 & $2.5 \%$ & $2.5 \%$ & $2.4 \%$ \\
\hline
\end{tabular}

Fuente: Elaboración propia con base en datos del Banco Mundial, World Development Indicators.

Gráfica 1. Análisis de Convergencia Sigma entre Estados Unidos, Canadá y México, 1960-2013

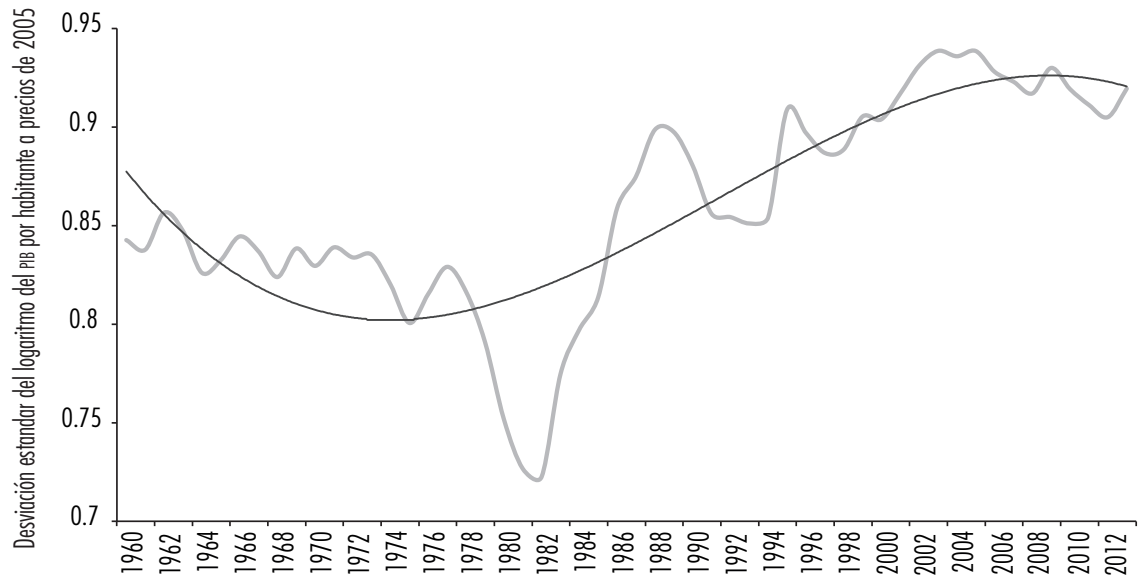

Fuente: Elaboración propia con base en datos del Banco Mundial, World Development Indicators.

Esta situación plantea un serio problema para los tres países, ya que el proceso de integración que se aceleró con la firma del TLCAN, se da entre sociedades cada vez más desiguales (Blecker y Esquivel, 2013); es decir, ocurre un mayor intercambio comercial e integración productiva, pero con crecientes niveles de desigualdad, ${ }^{3}$ lo que podría traducirse en un entorno de inestabilidad político-social a nivel regional. Suggestions for Implementation”, menciona que la apertura comercial no resulta completamente beneficiosa cuando se ignora la implementación de políticas sociales capaces de resarcir el daño que el aumento del comercio pudiera ocasionar, principalmente en el empleo. 


\section{LA PROPUESTA INICIAL: INCREMENTAR EL NIVEL DE COMERCIO EN LA REGIÓN}

La propuesta del TLCAN, en realidad, era reducir el esquema de protección arancelaria con el fin de alentar la creación productiva por la vía del comercio y la construcción de una zona de prosperidad compartida. Con este fin, se propuso una desgravación arancelaria progresiva para incrementar el comercio (Villarreal et al., 2013). Este proceso se realizó paulatinamente y dio lugar a un crecimiento espectacular del comercio. Las exportaciones de México a Estados Unidos se incrementaron de alrededor de 39 mil millones de dólares en 1993 a 299 mil millones de dólares en 2013, es decir, se multiplicaron por casi ocho veces; en tanto, las importaciones desde Estados Unidos pasaron de $41 \mathrm{mil}$ millones de dólares a 187 mil millones de dólares en el mismo periodo, lo que generó un superávit creciente. De esta forma, el déficit con el que México inició el TLCAN se transformó en un superávit que, de forma acumulada, alcanzó un billón de dólares.

El comercio con Canadá aun cuando creció en forma permanente -puesto que las exportaciones se multiplicaron por siete y las importaciones por 9.6 veces- no fue un proceso homogéneo como en el caso de Estados Unidos. Los intercambios comerciales con dicha nación han mostrado alzas y bajas, generándose déficits y superávits; sin embargo, ha registrado un déficit acumulado de 7 mil millones de dólares. En suma, el saldo de comercio exterior de la región es positivo para México, pero muestra que, en realidad, el proceso de integración se da efectivamente con Estados Unidos, pero en menor grado con Canadá.

El superávit con Estados Unidos permitió financiar los fuertes déficits que México observó con los países asiáticos y europeos. Si se considera el periodo de 1993 a 2013, el superávit de un billón de dólares fue insuficiente para cubrir el déficit que en ese mismo periodo México tuvo con Asia, Europa y Oceanía, que fue de 1.2 billones. En esta perspectiva, el perfil competitivo de las exportaciones mexicanas ocurre a nivel regional, pero se diluye en el marco de la globalidad.

En efecto, ocurrió una transformación de México como economía cerrada hacia una economía abierta, tal como se muestra en la gráfica 2 , en donde se aprecia que el grado de apertura de la economía medido como la relación de las exportaciones más importaciones sobre el PIB alcanzó 64\%, con lo cual se convirtió en una de las economías más abiertas del mundo. Si se compara esta situación con la prevaleciente en otros países, se observa que existe un proceso de creciente apertura comercial en México que no se observa en Es- 
Cuadro 2. México: comercio con los socios del TLCAN (miles de millones de dólares)

\begin{tabular}{|c|c|c|c|c|c|c|c|}
\hline \multicolumn{3}{|c|}{ Exportaciones } & \multicolumn{2}{|c|}{ Importaciones } & \multicolumn{3}{|c|}{ Saldo } \\
\hline Año & Canadá & EU & Canadá & EU & Canadá & EU & Con TLCAN \\
\hline 1993 & 1.5 & 39.0 & 1.0 & 41.4 & 0.4 & -2.5 & -2.0 \\
\hline 1994 & 1.5 & 51.6 & 1.6 & 54.8 & -0.1 & -3.2 & -3.3 \\
\hline 1995 & 2.0 & 66.3 & 1.4 & 53.9 & 0.6 & 12.4 & 13.0 \\
\hline 1996 & 2.2 & 80.6 & 1.7 & 67.5 & 0.4 & 13.0 & 13.5 \\
\hline 1997 & 2.2 & 94.4 & 2.0 & 82.0 & 0.2 & 12.4 & 12.6 \\
\hline 1998 & 1.4 & 93.9 & 2.0 & 84.9 & -0.6 & 9.0 & 8.3 \\
\hline 1999 & 2.3 & 120.3 & 2.9 & 105.3 & -0.6 & 15.0 & 14.4 \\
\hline 2000 & 3.3 & 147.4 & 4.0 & 127.5 & -0.7 & 19.9 & 19.2 \\
\hline 2001 & 3.1 & 140.6 & 4.2 & 113.8 & -1.2 & 26.8 & 25.6 \\
\hline 2002 & 3.0 & 141.9 & 4.5 & 106.6 & -1.5 & 35.3 & 33.9 \\
\hline 2003 & 3.0 & 144.3 & 4.1 & 105.4 & -1.1 & 38.9 & 37.9 \\
\hline 2004 & 3.3 & 164.5 & 5.3 & 110.8 & -2.0 & 53.7 & 51.7 \\
\hline 2005 & 4.2 & 183.6 & 6.2 & 118.5 & -1.9 & 65.0 & 63.1 \\
\hline 2006 & 5.2 & 211.8 & 7.4 & 130.3 & -2.2 & 81.5 & 79.3 \\
\hline 2007 & 6.5 & 223.1 & 8.0 & 139.5 & -1.5 & 83.7 & 82.2 \\
\hline 2008 & 7.1 & 233.5 & 9.4 & 151.3 & -2.3 & 82.2 & 79.8 \\
\hline 2009 & 8.2 & 185.1 & 7.3 & 112.4 & 0.9 & 72.7 & 73.6 \\
\hline 2010 & 10.7 & 238.7 & 8.6 & 145.0 & 2.1 & 93.7 & 95.8 \\
\hline 2011 & 10.7 & 274.4 & 9.6 & 174.4 & 1.0 & 100.1 & 101.1 \\
\hline 2012 & 10.9 & 287.8 & 9.9 & 185.1 & 1.0 & 102.7 & 103.8 \\
\hline 2013 & 10.5 & 299.4 & 9.8 & 187.3 & 0.6 & 112.2 & 112.8 \\
\hline
\end{tabular}

Fuente: Elaboración propia con información de BEA y Banco de México. 
Gráfica 2. Grado de apertura comercial, 1960-2013

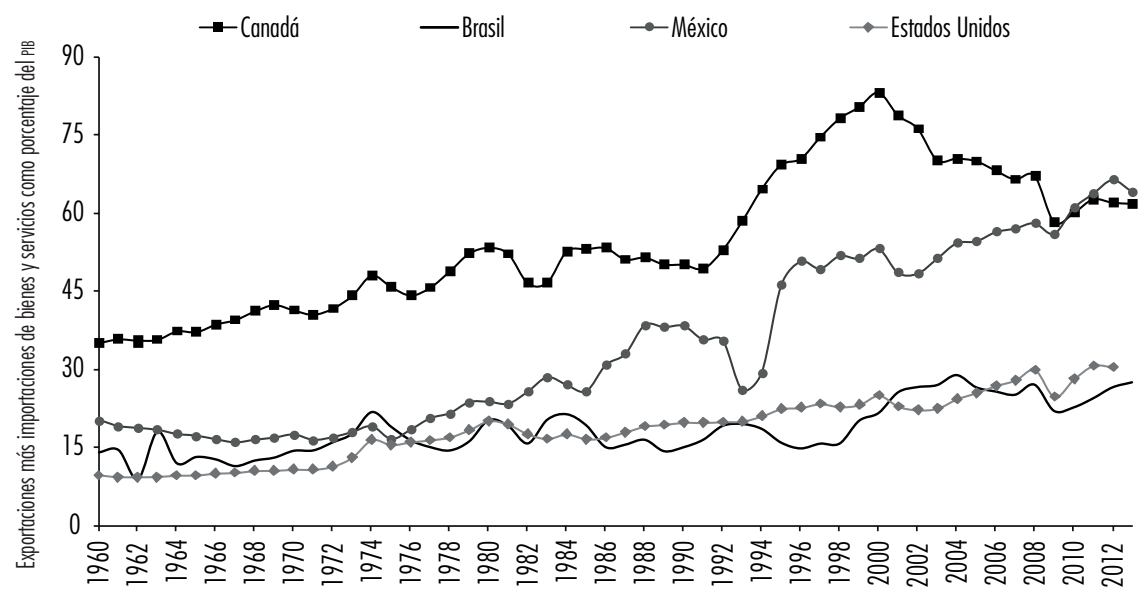

Fuente: Elaboración propia con base en datos del Banco Mundial, World Development Indicators.

tados Unidos -cuyo grado de apertura es de 30\%-, ni en otros países como Brasil -con un grado de apertura es de $28 \%$. En este sentido, el país apostó por la apertura sin cuidar las bases internas de la acumulación, esenciales para generar economías de escala y, por lo mismo, el fundamento para competir en la economía internacional.

Este impresionante desempeño de las exportaciones mexicanas estuvo fundamentado en la expansión de ciertos productos básicos. Tan sólo cinco rubros de exportación aportaron el $49 \%$ de las exportaciones: vehículos de motor, partes de vehículos de motor, petróleo y gas, equipos de cómputo, y equipos de audio y video. A pesar de que la mayor parte son productos manufacturados, las exportaciones de petróleo y gas continúan siendo importantes, puesto que representan el $11 \%$ de las exportaciones mexicanas hacia Estados Unidos (Villarreal et al., 2013). Sin embargo, la plataforma manufacturera ha sido el eje alrededor del cual se ha consolidado el TLCAN, y uno de los principales factores que han inducido una transformación productiva en México. 
Cuadro 3. Comercio de Estados Unidos con los socios del TLCAN, por tipo de productos, 2013 (miles de millones de dólares)

Socio TLCAN Exportaciones de Estados Unidos Importaciones de Estados Unidos

\begin{tabular}{llll}
\hline $\begin{array}{l}\text { Principales productos } \\
\text { (Clasificación SCIAN a 4 dígitos) }\end{array}$ & Valor & & $\begin{array}{l}\text { Principales productos } \\
\text { (Clasificación SCIAN a 4 digitos) }\end{array}$
\end{tabular}

\begin{tabular}{|c|c|c|c|c|}
\hline Canadá & Partes de vehículos de motor & 26.5 & Petróleo y gas & 87.6 \\
\hline & Vehículos de motor & 26.2 & Vehículos de motor & 44.5 \\
\hline
\end{tabular}

Productos derivados del petróleo y carbón $\quad 14.9 \quad$ Productos derivados del petróleo y carbón

Maquinaria para la agricultura

11.9 Partes de vehículos de motor

y la construcción

9.5 Procesamiento de metales no ferrosos

Otra maquinaria de uso general

$211.2 \quad$ Otros productos

Otros productos

(1)

Total de exportaciones a Canadá

300.2 Total de importaciones de Canadá

332.1

México Partes de vehículos de motor

21.1 Vehículos de motor

40.1

Productos derivados del petróleo

19.3 Partes de vehículos de motor

y carbón

Equipos de cómputo

14.8 Petróleo y gas

Semiconductores y otros componentes

13.0 Equipos de cómputo

electrónicos

Productos químicos básicos

10.1 Equipos de audio y vídeo

Otros productos

$147.9 \quad$ Otros productos

Total de exportaciones a México

226.2 Total de importaciones de México

280.5

Fuente: U.S. Bureau of Economic Analysis. 


\section{UNA TRANSFORMACIÓN PRODUCTIVA FUNDAMENTADA EN LA INVERSIÓN EXTRANJERA}

La transformación de México en un país altamente exportador estuvo apoyado en la inversión extranjera, la cual provino fundamentalmente de Estados Unidos, que incrementó su posición de inversión en México de 15 mil a 102 mil millones de dólares entre 1993 y 2012 (Villarreal et al., 2013), significando un multiplicador de 6.6 veces. Sin embargo, la inversión que realizó Estados Unidos en Canadá desde la firma del Tratado partió de una base mayor de casi 70 mil millones de dólares y se multiplicó por cinco veces, alcanzando los 351 mil millones de dólares en 2012. Durante el mismo periodo, La posición neta de la inversión estadounidense en México -es decir, la inversión extranjera de Estados Unidos en México menos la inversión de México en Estados Unidos- pasó de 14 mil a 86 mil millones de dólares, mientras que la posición de Canadá pasó de 29 mil a 126 mil millones de dólares. Esta mayor intensidad de la inversión estadounidense en territorio canadiense muestra que la relación no ha sido simétrica, comportamiento que también se observa en los mayores niveles de comercio entre Estados Unidos y Canadá respecto a los realizados con México, puesto que las exportaciones canadienses a Estados Unidos son superiores a las mexicanas en 50 mil millones de dólares. el antecedente del Acuerdo de Libre Comercio entre ambos países, signado en 1988. En este sentido, podemos atribuir que las relaciones no hayan sido simétricas debido a que tenían integraciones con distintos grados de madurez. 
Cuadro 4. TLCaN: Inversión extranjera directa según destino, 1993-2012 (millones de dólares)

\begin{tabular}{|c|c|c|c|c|}
\hline Año & IED de Canadá en EU & IED de EU en Canadá & IED de México en EU & IED de EU en México \\
\hline 1993 & 40,373 & 69,922 & 1,244 & 15,221 \\
\hline 1994 & 41,219 & 74,221 & 2,069 & 16,968 \\
\hline 1995 & 45,618 & 83,498 & 1,850 & 16,873 \\
\hline 1996 & 54,836 & 89,592 & 1,641 & 19,351 \\
\hline 1997 & 65,175 & 96,626 & 3,100 & 24,050 \\
\hline 1998 & 72,696 & 98,200 & 2,055 & 26,657 \\
\hline 1999 & 90,559 & 119,590 & 1,999 & 37,151 \\
\hline 2000 & 114,309 & 132,472 & 7,462 & 39,352 \\
\hline 2001 & 92,420 & 152,601 & 6,645 & 52,544 \\
\hline 2002 & 92,529 & 166,473 & 7,829 & 56,303 \\
\hline 2003 & 95,707 & 187,953 & 9,022 & 56,851 \\
\hline 2004 & 125,276 & 214,931 & 7,592 & 63,384 \\
\hline 2005 & 165,667 & 231,836 & 3,595 & 73,687 \\
\hline 2006 & 165,281 & 205,134 & 5,310 & 82,965 \\
\hline 2007 & 201,924 & 250,642 & 8,478 & 91,046 \\
\hline 2008 & 168,746 & 246,483 & 8,420 & 87,443 \\
\hline 2009 & 188,943 & 265,326 & 11,111 & 82,286 \\
\hline 2010 & 188,943 & 289,535 & 11,267 & 84,288 \\
\hline 2011 & 210,864 & 318,964 & 13,763 & 91,402 \\
\hline 2012 & 225,331 & 351,460 & 14,883 & 101,030 \\
\hline
\end{tabular}

Fuente: U.S. Bureau of Economic Analysis. 


\section{INTEGRACIÓN PRODUCTIVA DE LA REGIÓN DE AMÉRICA DEL NORTE: SURGIMIENTO DE LAS CADENAS DE VALOR}

La parte más positiva de la relación de México con los miembros del TLCAN ha sido, sin lugar a dudas, el desarrollo de cadenas productivas de valor, mismas que dominan hoy a la economía mundial. Las cadenas de valor a nivel global han sufrido transformaciones a lo largo de los últimos 20 años: a mediados de los años noventa, las cadenas globales de valor eran dominadas por Europa y por los miembros del TLCAN, cuestión que subsiste hasta la fecha, pero en forma declinante; en tanto los países del sudeste asiático, China y los países emergentes de Brasil, Rusia, India, Indonesia, Australia y Taiwán son los que progresivamente han ido creciendo en su participación, tal como puede observarse en la gráfica 3. En esta perspectiva, la firma del TLCAN ocurrió en un momento oportuno para integrarse a estas cadenas, ya que al reducirse las barreras arancelarias se pudo insertar en procesos de producción complejos y dinámicos, con un sistema de proveeduría espacialmente dispersa, en donde México logró incorporarse exitosamente en las industrias automotriz, electrónica y de productos químicos.

Gráfica 3. Participación Regional en las Cadenas Globales de Valor

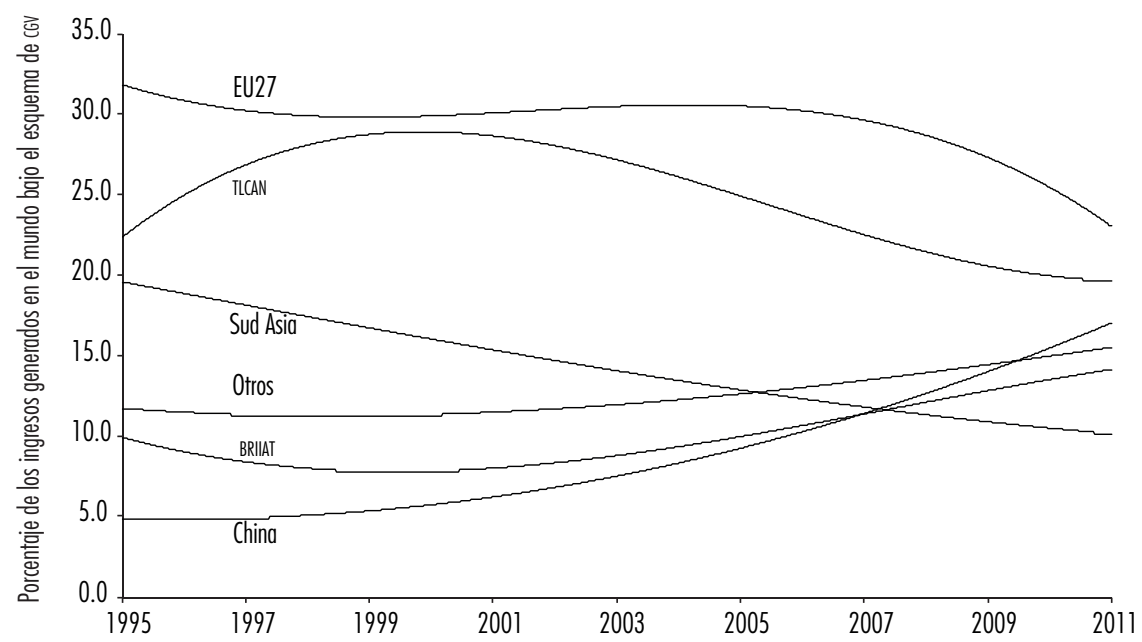

Nota: Las líneas mostradas corresponden a regresiones polinomiales de segundo orden.

${ }^{1}$ BRIIAT: Incluye Brasil, Rusia, India, Indonesia, Australia y Turquía.

Fuente: World Input-Output Database (WIOD). 
La incorporación a esta nueva dinámica de producción resultó en un proceso de modernización de la planta productiva mexicana. Sin embargo, fue difícil pasar de ser un centro ensamblador, a ser un integrante de las cadenas de valor. Esto implicó el desarrollo progresivo de proveedores locales o la atracción de proveedores foráneos para que se establecieran en el país, a fin de abastecer a los armadores de la industria automotriz y de la industria eléctrica-electrónica. Indudablemente el proceso no fue homogéneo, sino que fue moldeado por la intervención empresarial que ocurrió en cada sector. Como resultado, ciertas cadenas se tornaron más complejas, mientras que en otras se observaron retrocesos.

En las gráficas 4, 5 y 6 se muestra la forma en que los países del Tratado enfrentaron el proceso de integración a las cadenas de valor, considerando información para el periodo de 1995 a 2009. Conviene señalar que el análisis mostrado se realizó para cada país y que incorpora una serie de tendencias: en primer lugar, el crecimiento o decrecimiento de la aportación nacional al valor agregado de lo exportado por el país al que se hace referencia, y en segundo, la forma en que aportan los otros dos miembros del Tratado al valor agregado de las exportaciones del mismo país; en este sentido, tenemos un análisis tridimensional que permite observar las disparidades entre estas tendencias 5

Con el objetivo de conocer el papel que cada país desempeña, se utilizó la base de datos elaborada por la OCDE en colaboración con la OMC, en la que se describen las relaciones comerciales entre países, por tipo de actividad económica. Este banco de datos permite descomponer el valor agregado de las exportaciones de un determinado país y en un determinado sector, por cada país y sector de origen. El periodo analizado corresponde de 1995 a 2009 por la disponibilidad de información de esta fuente de datos. 
Gráfica 4. México: variación en la participación porcentual en la incorporación de VA en el total de las exportaciones, 1995-2009 (por país de origen y fuente de la industria)

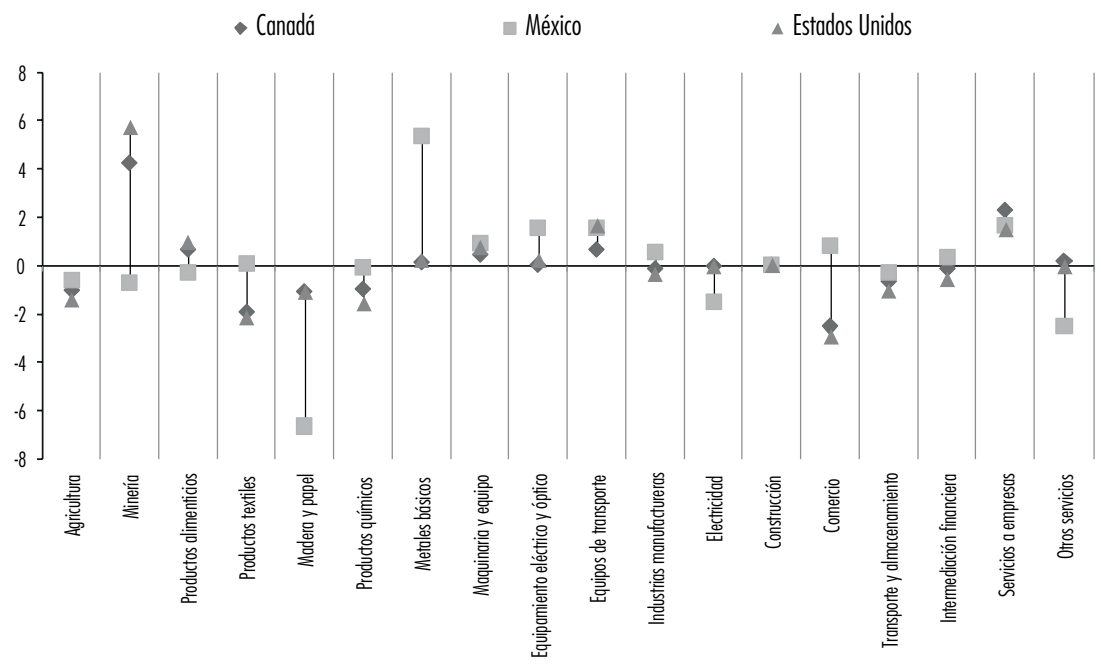

Fuente: Elaboración propia con información de 0ECD-WTO Trade in Value Added Database (TiVA).

Gráfica 5. Canadá: variación en la participación porcentual en la incorporación de va en el total de las exportaciones, 1995-2009 (por país de origen y fuente de la industria)



Fuente: Elaboración propia con información de OECD-WTO Trade in Value Added Database (TiVA). 
Gráfica 6. Estados Unidos: variación en la participación porcentual en la incorporación de va en el total de las exportaciones, 1995-2009 (por país de origen y fuente de la industria)

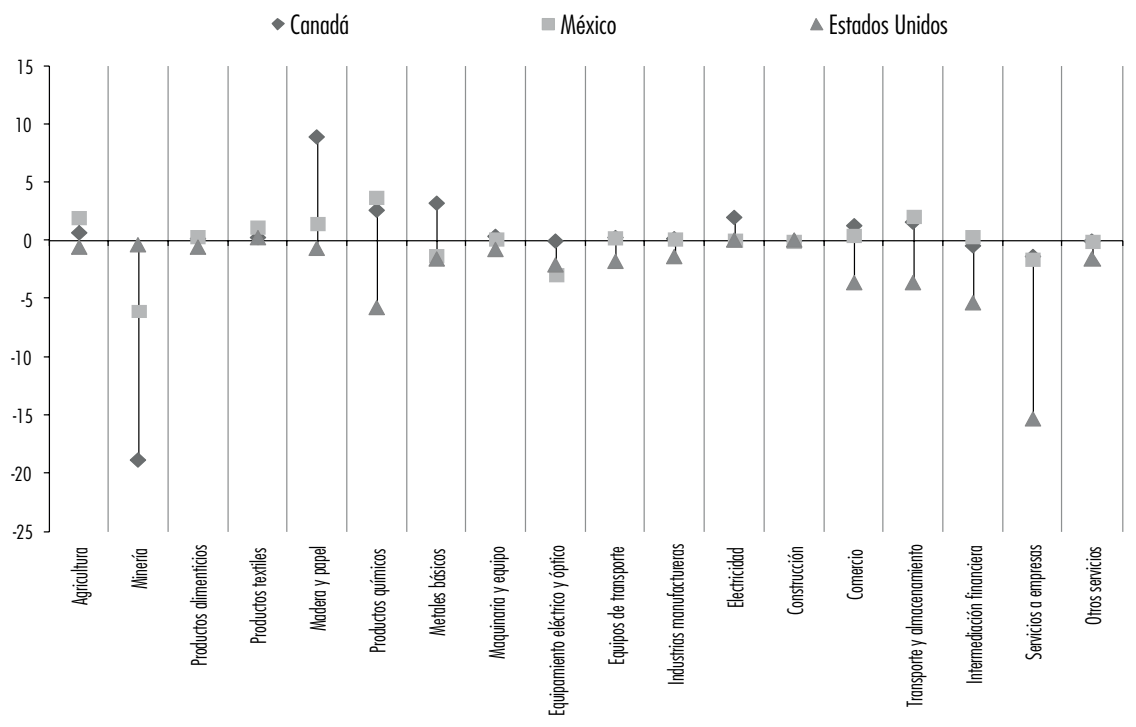

Fuente: Elaboración propia con información de OECD-WTO Trade in Value Added Database (TiVA).

Considerando lo anterior, el ejemplo más representativo para el caso de México es el de la minería, donde Canadá y Estados Unidos elevan su aportación al valor agregado de las exportaciones mexicanas, y que contrasta con la disminución observada en las exportaciones del sector comercio. Podemos observar ciertas tendencias en la generación de valor agregado doméstico a las exportaciones de México:

- Sectores donde México redujo la incorporación del valor agregado en el total de exportaciones: agricultura, minería, productos alimenticios, madera y papel, transporte y almacenamiento y otros servicios.

- Sectores donde México mantuvo la incorporación del valor agregado en el total de exportaciones: productos textiles, productos químicos, e intermediación financiera.

\footnotetext{
Beatty (2012) argumenta que la integración del mercado mexicano y canadiense promovió inversiones en la explotación minera en diferentes regiones del país, así como el desarrollo de otras industrias como la aeronáutica, a través de la empresa Bombardier.
} 
- Sectores donde México aumentó la incorporación del valor agregado en el total de las exportaciones: metales básicos, maquinaria y equipo, equipamiento eléctrico y óptico, equipo de transporte, comercio, y servicios a empresas.

Si se analiza la situación para los otros miembros del TLCAN, se observa que en el comercio intrarregional sí existe un desplazamiento que benefició a México, ya que precisamente en aquellos sectores en donde México aumenta su contenido de valor agregado, Canadá y Estados Unidos lo reducen, como es el caso en minería, productos químicos, equipos eléctrico y óptico. En algunos casos, las gráficas nos muestran que en ciertos sectores, como servicios a empresas, ocurre una reducción en la participación de los tres países, lo que se explica por la incorporación de valor extra-regional, en donde las empresas europeas se han especializado en esta área y están sacando ventajas de ello.

Dentro de las cadenas de valor, el caso más paradigmático ha sido el del sector automotriz, actividad en la que México ha desarrollado un clúster sumamente competitivo, convirtiéndose en el eje de la renovación de la industria automotriz de América del Norte. Como señala el reporte de Ángeles Villareal y de Ian Fergusson (2013):

El TLCAN fue fundamental en la integración de la industria automotriz de América del Norte, que ha experimentado algunos de los cambios más significativos en el comercio tras el acuerdo. Los fabricantes de autopartes estadounidenses pueden usar los insumos y componentes producidos por otro socio del TLCAN para construir autopartes, que luego son enviadas a otro país del TLCAN para ensamblarlas en un vehículo que se vende en cualquiera de los tres países del TLCAN. El tratado consistió en una gradual eliminación de los aranceles y la eliminación de muchas de las barreras no arancelarias al comercio, preveía disposiciones de origen uniformes, una mayor protección de los derechos de propiedad intelectual, la adopción de prácticas de contratación pública menos restrictivas, y la eliminación de los requerimientos de desempeño a los inversores de otros países del TLCAN. El tratado establece la eliminación de las restrictivas políticas de comercio e inversión de México y la eliminación de los aranceles estadounidenses sobre automóviles y autopartes.

El acelerado desarrollo de la industria automotriz se puede observar en el cuadro 5, donde se aprecia cómo México incrementó sus exportaciones al mercado estadounidense en $587 \%$, en tanto las importaciones aumentaron en $245 \%$. Conviene señalar que Canadá se quedó rezagado, lo que muestra 
el papel tan relevante que jugó México en esta transformación productiva de la región.

Desafortunadamente este proceso tan dinámico no se dio en forma generalizada, y ocasionó el declive de muchas industrias que habían sido paradigmáticas en el país -como la textil, la del vestido y la del zapato, entre otras-y que generaban mayor empleo en el país.

Cuadro 5. Estados Unidos: el comercio de vehículos y autopartes, 1993-2013 (miles de millones de dólares)

\begin{tabular}{|c|c|c|c|c|c|c|c|c|}
\hline & \multicolumn{3}{|c|}{1993} & \multicolumn{3}{|c|}{2013} & \multicolumn{2}{|c|}{$\begin{array}{l}\text { \% Variación } \\
\text { 1993-2013 }\end{array}$} \\
\hline & 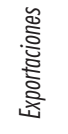 & 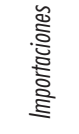 & 홍 & 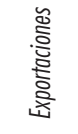 & 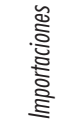 & 홍 &  & 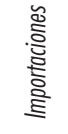 \\
\hline \multicolumn{9}{|l|}{ México } \\
\hline Vehículos & 0.2 & 3.7 & 3.9 & 4.8 & 40.1 & 44.9 & $2300 \%$ & $984 \%$ \\
\hline Autopartes & 7.3 & 7.4 & 14.7 & 21.1 & 36.2 & 57.3 & 189\% & $389 \%$ \\
\hline Total & 7.5 & 11.1 & 18.6 & 25.9 & 76.3 & 102.2 & $245 \%$ & $587 \%$ \\
\hline \multicolumn{9}{|l|}{ Canadá } \\
\hline Vehículos & 8.2 & 26.7 & 34.9 & 26.2 & 44.5 & 70.7 & $220 \%$ & $67 \%$ \\
\hline Autopartes & 18.2 & 10.3 & 28.5 & 26.5 & 13.5 & 40 & $46 \%$ & $31 \%$ \\
\hline Total & 26.4 & 37 & 63.4 & 52.7 & 58 & 110.7 & $100 \%$ & $57 \%$ \\
\hline \multicolumn{9}{|l|}{ Mundo } \\
\hline Vehículos & 18.9 & 63 & 81.9 & 73.1 & 175.7 & 248.8 & $287 \%$ & $179 \%$ \\
\hline Autopartes & 33.4 & 38.3 & 71.7 & 60.6 & 100.4 & 161 & $81 \%$ & $162 \%$ \\
\hline Total & 52.3 & 101.3 & 153.6 & 133.7 & 276.1 & 409.8 & $156 \%$ & $173 \%$ \\
\hline
\end{tabular}

Nota: El scian es el estándar utilizado por las agencias estadísticas federales en la clasificación de los establecimientos comerciales con el fin de recopilar, analizar y publicar datos estadísticos relacionados con la economía de las empresas de Estados Unidos.

Fuente: Congressional Research Services a partir de datos comerciales de la USITC en http://dataweb.usitc.gov. Para 2013, "vehículos" se compone de elementos bajo el Sistema de Clasificación Industrial de América del Norte (SCIAN) número 3361 y "Autopartes" se compone de elementos bajo NAIC número 3363. 


\section{LA HIPÓTESIS DE CRECIMIENTO DEL EMPLEO CON MEJORES REMUNERACIONES}

Las premisas esbozadas en el discurso político sobre el Tratado suponían que el crecimiento del comercio y el desarrollo industrial generarían efectos positivos sobre el resto de la economía, elevando el empleo y los salarios. Sin embargo, el proceso de crecimiento del empleo en México no fue el esperado. ${ }^{7}$ El crecimiento promedio de la población económicamente activa desde la puesta en marcha del Tratado fue de $2.2 \%$, provocando que pasara de 35.4 millones de personas en 1994 a 52.8 millones en 2014, es decir, un incremento de 17 millones de personas adicionales con todas las prestaciones de ley. Sin embargo, no fue posible generar este nivel de empleo debido a que la mayoría de las actividades cuentan con bajos efectos multiplicadores, tal como puede observarse en el cuadro 6, donde se indica que el nivel del multiplicador promedio es de 1.47 por unidad de valor bruto de la producción. Si se analiza con detenimiento el vector de multiplicadores de empleo, se observa que las actividades en donde se centró la atención para el desarrollo de cadenas de valor del tratado, tienen multiplicadores por abajo del promedio, generando poco empleo: la industria de fabricación de equipo de transporte tiene un multiplicador de 1.32, y el de fabricación de equipo de cómputo y de accesorios electrónicos es de 1.05. En esta perspectiva, al no generarse el número de empleos de calidad, el país dejó que creciera el empleo informal y la migración como mecanismos de ajuste.

Estos multiplicadores de empleo permitieron que se crearan 2.3 millones de empleos en el sector manufacturero, incrementándose el empleo de esta actividad en 46\%. Esta dinámica, sin embargo, no logró resolver el problema del fuerte crecimiento de la oferta de empleo en el periodo, que fue de 17 millones de personas. Una cuestión importante en este entorno del empleo manufacturero es que en tanto México creó empleo en dicho sector, en Estados Unidos se redujo en 4.6 millones y llevó a que se arguyera que Detroit se había trasladado al sur de la frontera. Esta afirmación es parcialmente cierta, porque en realidad se destruyó más empleo en Estados Unidos que el que se creó en México, por lo que muchas de las plazas manufactureras en realidad migraron hacia otras regiones, especialmente Asia.

Polaski (2004) menciona que el acuerdo comercial atrajo empleos, principalmente en el sector manufacturero que se fue instalando en México. Sin embargo, el empleo en el sector primario se vio afectado a partir de la creciente importación de alimentos provenientes de Estados Unidos. 
Cuadro 6. Muttiplicadores de empleo por subsector de actividad económica, 2008

Subsectores de actividad económica con mayor multiplicador de empleo

Generación, transmisión y suministro de energía eléctrica

Compañías de fianzas, seguros y pensiones

Servicios relacionados con las actividades agropecuarias y forestales

Fabricación de productos derivados del petróleo y del carbón

Servicios de almacenamiento

Industria alimentaria

Transporte aéreo

Industria química

Servicios relacionados con la minería

Ganadería

Fabricación de equipo de transporte

Subsectores de actividad económica con menor multiplicador de empleo

Fabricación de equipo de generación eléctrica y aparatos eléctricos

Aprovechamiento forestal

Servicios artísticos y deportivos y otros servicios relacionados

Extracción de petróleo y gas

Servicios educativos

Servicios inmobiliarios

Fabricación de equipo de computación, comunicación y medición

Fabricación de prendas de vestir

Creación y difusión de contenido de internet

Servicios de alquiler de marcas registradas, patentes y franquicias

Fuente: Elaboración propia con información INEGI, Matriz de Insumo-Producto 2008. 
Junto a la baja creación de empleo de calidad, se dio un proceso de deterioro del salario real en México, cuestión que se refleja en el hecho de las remuneraciones promedio de los trabajadores manufactureros en México son tan sólo el $17.8 \%$ de los prevalecientes en Estados Unidos. Lo anterior contrasta con lo que aconteció en Canadá, donde los salarios se ajustaron a lo largo de los 20 años del Tratado, pero que actualmente son superiores a los estadounidenses. Este diferencial en las remuneraciones es lo que ha provocado que la desigualdad entre los países no logre reducirse, ya que al no elevarse los niveles de ingreso en el caso mexicano a la par que los estadounidenses y los canadienses, el PIB por habitante se ha mantenido rezagado (Blecker y Esquivel, 2013); es decir, es un problema de productividad y de una política fundamentada en bajos salarios en México, en donde se decidió congelar el salario mínimo en los años ochenta y desde entonces se ha mantenido rezagado.

Esta política instrumentada en México ha provocado una estrechez del mercado interno mexicano, pues las bajas remuneraciones han resultado en niveles de consumo limitados y esto, a su vez, ha reducido el potencial del consumo de masas, que es parte fundamental del modelo de crecimiento en los capitalismos modernos. Para ver el rezago del consumo se estimaron los gastos de consumo de las familias en el periodo 1993 a 2012 para los tres países, encontrándose que crecieron a una tasa de $1.9 \%$ en Estados Unidos, de 2\% en Canadá y de sólo 1.4\% en México.

Grática 7. Remuneración por hora de los trabajadores manufactureros como porcentaje de la remuneración en Estados Unidos, 1995-2012

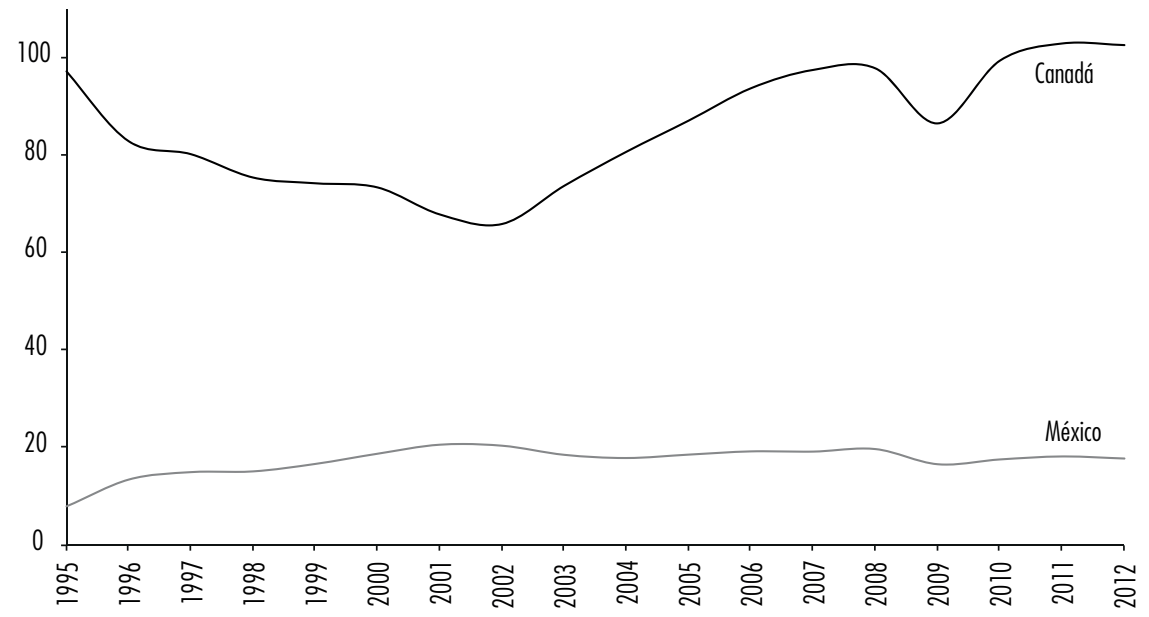

Fuente: U.S. Bureau of Labor Statistics. 


\section{REFLEXIONES HACIA EL FUTURO: LA NECESIDAD DE UNA AGENDA PARA EL SIGLO XXI}

Los 20 años transcurridos del TLCAN han iniciado un proceso de integración productiva entre los tres países que lo integran. Una construcción transnacional de este tipo requiere de décadas, por lo que se debe realizar una evaluación conjunta para ver los puntos positivos y negativos de esta relación, que vaya más allá de lo comercial, y que busque construir una nueva sociedad progresivamente integrada. Una sociedad de este tipo requiere de mayores inversiones conjuntas, para equipararse a lo que se ha realizado en otros procesos de integración en el mundo, tal como ha acontecido en la Unión Europea. En la trayectoria de largo plazo (1960 a 2013), el nivel de formación bruta de capital de México ha sido la más baja, con 19.6\% del PIB, seguido por Estados Unidos con $21 \%$, y por Canadá con $22 \%$. Para transformar a la región en un área de coprosperidad es necesario elevar este nivel de acumulación de capital para igualar las condiciones de vida entre los tres países.

Gráfica 8. Una región con baja acumulación de capital, 1960-2013



Fuente: Banco Mundial, World Development Indicators database. 
Una agenda para elevar la inversión en forma conjunta permitiría fijar áreas estratégicas, en las cuales se realizarían proyectos de inversión para incrementar la infraestructura necesaria que iguale las condiciones prevalecientes en los tres países del TLCAN, y lograr con ello elevar la competitividad ante el reto que plantea el nuevo Acuerdo Estratégico Trans-Pacífico de Asociación Económica, región donde los países asiáticos - como Corea, China y Taiwánmantienen coeficientes de inversión superiores al 30\% de su PIB. Esta propuesta estaría ligada de igual forma a aquellos sectores en que la integración de cadenas productivas representa una ventaja global.

Otro elemento clave en esta agenda del siglo XXI será crear condiciones para desarrollar a América del Norte como una sociedad de innovación creativa, para lo que se requiere elevar de forma homogénea la calidad del capital humano. Para alcanzar este fin, se debe construir un esquema de planeación conjunta para elevar el promedio de escolaridad y facilitar los procesos de certificación de los programas de educación. Este proceso conllevaría a facilitar el intercambio de experiencias en materia de investigación, lo que debería estar acompañado de mecanismos de financiamiento para realizar desarrollos conjuntos; en este sentido, sería deseable fusionar las instancias que impulsan actualmente la investigación y el desarrollo, que como se comentó en la reunión de presidentes de América del Norte en 2014 (Toluca, México), podrían derivar en un Consejo Trilateral de Investigación, Desarrollo e Innovación (Presidencia de la República, 2014).

Un punto clave de la agenda para el siglo XXI del TLCAN será establecer un esquema de colaboración en materia de energía, pensando en que la región se convertirá en una zona de energía renovable para el año 2050, sin contar con problemas de abastecimiento de combustibles, buscando lograr la autosuficiencia energética. En este sentido, debería fijarse la meta de transformar a los países del TLCAN en una región de economía verde, para reducir los efectos del cambio climático que hoy ponen en riesgo a diferentes zonas de América del Norte.

Todos estos elementos, si bien claves para una agenda XXI del TLCAN, son insuficientes para reducir en un tiempo razonable la desigualdad. Se requiere un gran acuerdo para poder acotar la desigualdad, y para ello es necesario un compromiso amplio entre las sociedades de América del Norte con el fin de establecer un mecanismo que impulse el desarrollo de las zonas con mayor marginación. La región tiene que discutir la mejor forma de establecer mecanismos compensatorios que eleven el nivel de vida de estas zonas, que no son exclusivas de México, sino que se encuentran también en Estados Unidos y Canadá. Sin lugar a dudas, esto es un tema difícil para sociedades en donde, 
por el momento, no existe ningún proceso de esta naturaleza. La intención sería buscar que los ingresos por habitante de la región tendieran a convergir a lo largo del siglo xxi.

\section{AGRADECIMIENTOS}

Agradezco al PAPIIT, en particular al proyecto IN304514 "El desarrollo empresarial en las variedades de capitalismo", por el apoyo brindado para el desarrollo de esta investigación.

\section{BIBLIOGRAFÍA}

Beatty, Perrin and Andrés Rozental (2012), Forging a new Strategic PartnerShiP between canada and Mexico Special RepoRt, The Centre for International Governance Innovation and the Canadian Chamber of Commerce.

Blecker, Robert A. (2005), "The North American Economies after NAFTA: A Critical Appraisal", International Journal of Political Economy, vol. 33, núm. 3.

Blecker, Robert A. and Gerardo Esquivel (2013), "Trade and the Development Gap", in Peter H. Smith and Andrew Selee, Mexico and the United States: The Politics of Partnership, Boulder, co, Lynne Rienner.

Calva, J. L. (2004), "Ajuste estructural y TLCAN: efectos en la agricultura mexicana y reflexiones sobre el ALCA", El Cotidiano, vol. 19, núm. 124, marzoabril, pp. 14-22, UAM, México.

Capaldo, Jeronim (2014), "Trade Hallucination: Risks of Trade Facilitation and Suggestions for Implementation”, GDAE Working Paper, pp. 14-02.

Feils, Dorothee J. and Manzur Rahman (2008), "Regional Economic Integration and Foreign Direct Investment: The Case of NAFTA", MIR: Management International Review, vol. 48, núm. 2, pp. 147-163.

Kuznets, Simon (1956), "Quantitative Aspects of the Economic Growth of Nations: Levels and Variability of Rates of Growth", Economic Development and Cultural Change, vol. 5, núm. 1 (October), pp. 1, 5-94.

Moreno-Brid, J.C.; Santamaría, J. y Rivas, J.C. (2006), "Manufactura y TLCAN: un camino de luces y sombras", Economía UNAM, vol. 3, núm. 8, UnAM, México.

Presidencia de la República (2014), "América del Norte del siglo xxi: construyendo la región más dinámica y competitiva”, Acuerdos de reunión del 19 de febrero de 2014, México. 
Clemente Ruiz

Polasky, Sandra (2004), Mexican Employment, Productivity and Income a Decade after NAFTA, brief Submitted to the Canadian standing Senate Committee on Foreign Affairs.

Villarreal, M. Ángeles and Ian F. Fergusson (2013), NAFTA at 20: Overview and Trade Effects, Congressional Research Service. 